\title{
Der Weihnachtsengel
}

\section{Erica Brühlmann-Jecklin}

Psychotherapeutin SPV / Supervisorin, Schriftstellerin und Liedermacherin

Niemand wusste genau, was Monika noch wahrnahm. Sie lag auf den gelben Laken, die das Heim vor Jahresfrist anstelle der weissen eingeführt hatte, was den Angehörigen ihre schwierige Situation etwas erleichterte, wenn sie ihre Lieben so liegen sahen. Monika konnte seit langem nichts mehr sehen, weder Farben noch Formen, zu sehr hatte die bösartige Multiple-SkleroseForm nicht nur die Isolationsschicht jener Nerven zerstört, die der Bewegung dienen sollten, sondern auch die der Sehnerven. Das bisschen Licht, das bis vor kurzem noch auf ihre Sehzellen dringen konnte, blieb jetzt durch das permanente Geschlossenhalten der Augenlider auch weg.

So lag Monika da, als Folge eines siebenjährigen Leidens, das sie mehr und mehr zur Hilflosigkeit verdammt und ihr alles genommen hatte: Arbeit, Freiheit, Selbständigkeit. Und ganz banal: stehen, gehen, sitzen und sehen zu können. Als ihre Mutter sie den Pflegenden dieses Heims, dieser Station anvertrauen musste, schweren Herzens, weil die Kraft nicht mehr reichte, nach dem Enkel und der schwerbehinderten Tochter zu schauen, konnte Monika noch im Rollstuhl sitzen, skandierend Bedürfnisse aussprechen, zuweilen lallend auch ein paar kräftige Schimpfwörter, zu Recht wie einem schien, denn was war das noch für ein Leben. Aber Monika hing an diesem bisschen Leben, das ihr noch geblieben war.

Die Krankheit war über die junge Frau eingebrochen, drei Jahre nachdem sie den Bub geboren hatte, ihren kleinen Weihnachtsengel, wie sie gerne sagte, der drei Tage nach dem Weihnachtstag zur Welt gekommen war, den sie deshalb Angel nannte, und nachdem der Vater des Kindes aus eigener innerer Not die beiden verlassen hatte. Der Bub konnte sich nicht erinnern, die Mutter anders als behindert gekannt zu haben. Die beiden hingen aneinander, und die Grossmutter sorgte dafür, dass sie zusammenbleiben konnten, auch als die Krankheit gnadenlos zuschlug, Monat für Monat für neue böse Überraschungen sorgte, Monika unaufhaltsam Kraft um Kraft raubte.

Seit Monika in den gelben Leintüchern des Pflegeheims lag, bemühte sich die Grossmutter darum, dass Angel so oft wie möglich zur Mutter konnte. Der Weg führte vom kleinen Weiler im Bernbiet zum Dorf, von dort mit der Schmalspurbahn in die Stadt und von da weiter mit dem Bus ins Aussenquartier zum Heim. Zu Beginn noch, wenn sich die Tür ins Zimmer der Schwerbehin- derten öffnete, diese den Schritt der beiden erkannte, ging ein zartes Strahlen über ihr Gesicht, und sie sagte: «Angel, mein Weihnachtsengel.» Nicht nur in der Adventszeit, nein, das ganze Jahr hindurch.

Jetzt war Advent, und Angel würde bald seinen zehnten Geburtstag feiern. Zwei, drei Mal die Woche kam er mit der Grossmutter ins Heim, brachte Christrosen und Kerzen, die sie nicht anzünden durften, auch Tannenreisig, der etwas Weihnachtsduft ins Krankenzimmer brachte, und die Grossmutter sass auf der einen Seite des Bettes, und Angel auf der anderen, und er erzählte der Mutter alles, was ihm gerade einfiel.

«Vorgestern war die Katze im Keller eingesperrt, es ging lange, bis wir ihr Miauen hörten. Und Tobi möchte immer mitkommen, wenn wir zu dir kommen, er möchte dich auch besuchen, Mama, aber Hunde dürfen ja nicht.»

Monika reagierte immer weniger auf das Erzählen des Buben, er aber hielt daran fest, ungeachtet ihrer Reaktionen und Nichtreaktionen, ass zusammen mit der Grossmutter Mandarinen, so dass der Weihnachtsduft ganz deutlich wurde, und die Grossmutter presste einen Schnitz auf Monikas Lippen aus, damit sie teilhaben konnte an dieser besonderen Jahreszeit. Draussen schneite es, und Weihnachten kam mit jedem Tag näher. "An Heiligabend wollen wir bei ihr sein", hatte die Grossmutter gesagt, und so machten sie sich am Nachmittag des 24. Dezembers auf den Weg, ohne Blumen oder sonst einem Geschenk, denn Monika lag seit ein paar Tagen im Koma, wie die Pflegenden sagten. Unter einem Koma konnte sich Angel nichts vorstellen, wohl aber darunter, dass die Mutter jetzt vor allem schlafen wollte, so tief, dass sie nicht erwachte, wenn er und die Grossmutter an ihrem Bett sassen.

Sie treten durch den Haupteingang in den schlecht beleuchteten Korridor, vorbei an kleinen Christbäumen mit elektrischen Kerzen, zum Lift, in den ersten Stock, vorbei am mit Engelshaaren, Silberfäden und roten Kugeln geschmückten Stationszimmer, hinein durch die offenstehende Tür ins Zimmer der schlafenden Mutter. Sie setzen sich, wie immer die Grossmutter auf die eine Bettseite, Angel auf die andere. Monika liegt ruhig da, die Augen geschlossen. Angel schaut sie an. Blickt auf die gelben Tücher, auf die kurz geschnittenen Haare der Mutter, bemerkt den unruhigen Atem, nimmt ihre schlaff auf der Bettkante liegende Hand, hält sie mit seiner Kinderhand, beginnt leise zu sum- 
men. Die Grossmutter blickt aufmerksam durch die schimmernden Tränen, die ihr in die Augen treten, auf den Bub, der jetzt Worte zu singen beginnt, leise, innig:

"Leise rieselt der Schnee,

still und starr ruht der See,

weihnachtlich glänzet der Wald:

Freue dich, 's Christkind kommt bald.»

Angel spürt, wie sich Mutters Hand regen will. Wie ihr Mund sich ein wenig bewegt, etwas sagen möchte. «Sing weiter, Angel», sagt die Grossmutter.

«Drei Ängeli flüge dür d' Nacht eso wiit, sie flüge zum Chrippli wo's Jesus-Chind liit.

Das erscht bringt es Blüemli,

das zweit bringt en Schtärn,

das dritt singt es Liedli: I ha di so gärn.»

Mühsam wollen sich Monikas Lippen zu einem Wort formen, aber die Kraft reicht nicht. Ihr Atem wird ruhiger, tiefer, und draussen beginnt sich die Dämmerung über Häuser, Felder und den nahen Wald zu legen.

«Komm!», sagt die Grossmutter, «wir müssen heim, bevor es dunkel ist.»

«Schöne Weihnachten!», flüstert Angel zur Mutter, während er sich langsam erhebt, Mutters Hand los- lässt, seine in die der Grossmutter legt, mit ihr das Zimmer verlässt, das Heim, in dem jetzt die elektrischen Kerzen an den Bäumchen leuchten.

Ein kalter Wind weht und die Dunkelheit hatte sich auf den Weiler gelegt, als die beiden ins Haus treten, die Schuhe ausziehen, Jacke und Mantel an den Haken hängen, die Mützen auf die Kommode am Eingang legen. Noch sind sie nicht in der warmen Stube, als das Telefon klingelt. Die Grossmutter nimmt den Hörer, setzt sich, lauscht, nickt, legt wieder auf, zieht ihren Enkel zu sich, sagt: «Angel, deine Mama darf im Himmel Weihnachten feiern. Dort singt ein Chor von tausend Engeln und viele hundert Glocken läuten zur Geburt des Jesuskindes.» Angel setzt sich zur Grossmutter, legt den Kopf in ihren Schoss, weint, während ihm die Grossmutter sanft über die rotbraunen Haarlocken streicht, und sie tröstend zum Bub sagt: «Du hast ihr heute mit Deinem Singen das allerschönste Geschenk gegeben.»

Seither sind einige Jahre vergangen, aber Angel weiss, dass er bis heute Mutters Weihnachtsengel ist, und dass seit jenem Heiligabend über ihm ein ganz besonders lieber Engel wacht.

Bildnachweis

Jorisvo | Dreamstime.com

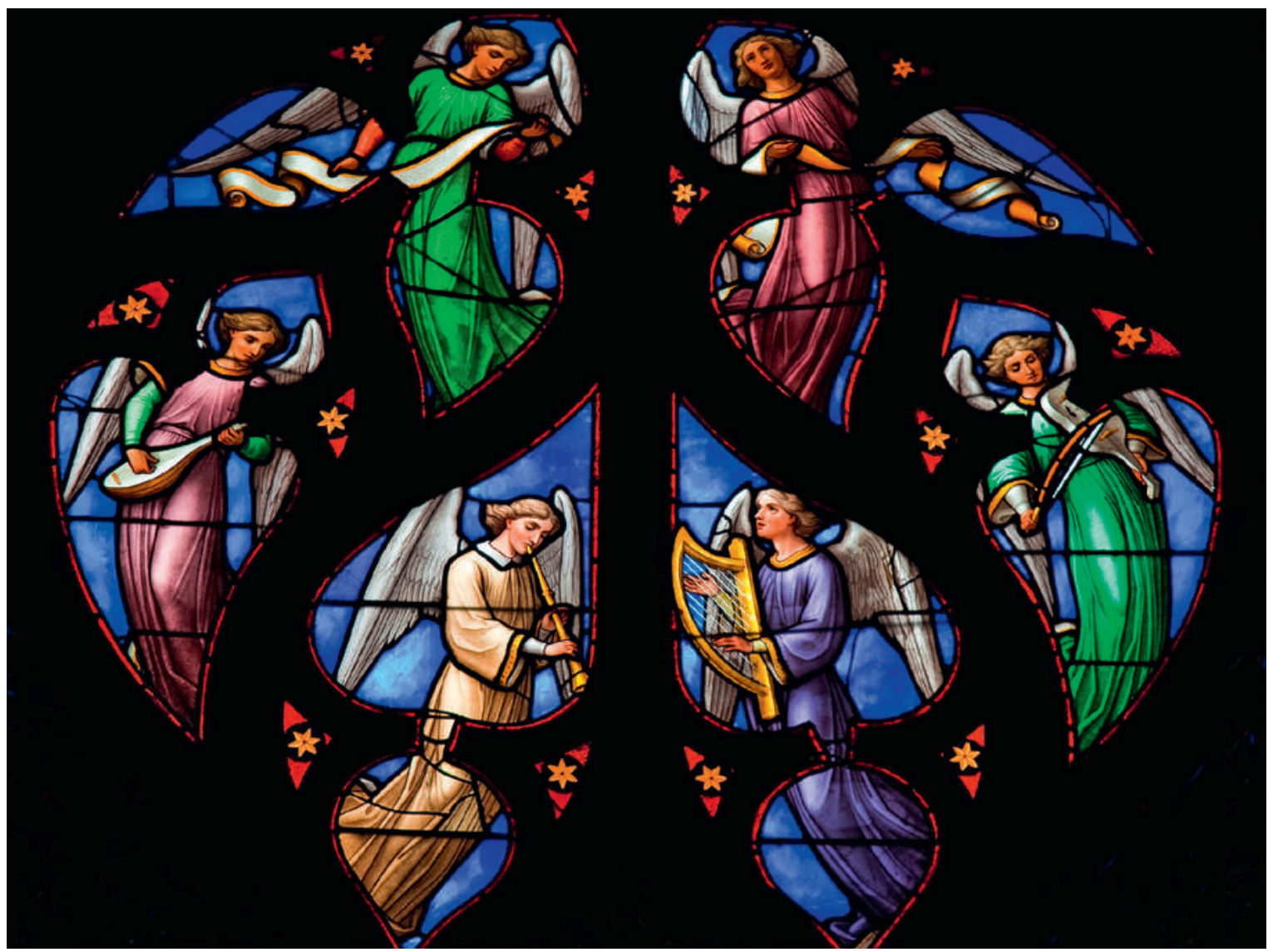

Dieses Jahr würde an Weihnachten ein Chor von Engeln für seine Mutter singen. 